\title{
Clinical and Spectrophotometric Evaluation of Skin Photoadaptation in Vitiligo Patients after a Short Cycle of NB-UVB Phototherapy
}

\author{
Mariachiara Arisi ${ }^{a}$ Maria Teresa Rossi ${ }^{a}$ Marta Fusano ${ }^{a}$ Alessandra Gualini ${ }^{a}$ \\ Cesare Tomasi $^{\mathrm{b}}$ Erica Moggio a Simone Caravelloa \\ Piergiacomo Calzavara-Pinton $^{\text {a }}$ Marina Venturinj ${ }^{a}$ \\ a Dermatology Department, University of Brescia, ASST Spedali Civili of Brescia, Brescia, Italy; bepartment of \\ Medical and Surgical Specialties, Radiological Sciences and Public Health, Occupational Health, University of \\ Brescia, Brescia, Italy
}

\section{Keywords}

Narrow-band ultraviolet B phototherapy - Photoadaptation . Spectrophotometry · Vitiligo

\begin{abstract}
Background: The phenomenon of photoadaptation to narrow-band ultraviolet B (NB-UVB) radiation has been previously described in vitiligo and has usually been clinically measured by the assessment of the minimal erythema dose (MED) after phototesting. Object/ves: To assess the photoadaptive response in vitiligo and healthy skin after NB-UVB phototherapy not only clinically, but also by spectrophotometry. Materials and Methods: Fourteen patients affected by generalized vitiligo underwent NB-UVB phototherapy twice weekly for 12 weeks. Before and after phototherapy, a phototesting procedure was administered on vitiligo patches and adjacent healthy skin with a solar simulated radiation (SSR). Visual assessment of the MED took place after $24 \mathrm{~h}$. A spectrophotometer was used to assess the $a^{*}$ value and the melanin index $\left.(\mathrm{M})^{*}\right)$, as signs of skin erythema and pigmentation. The photoadaptation factor (MED-PF) and the $a^{*}$ photoadaptation factor (a*-PF) were calculated. Resu/ts: After NB-UVB phototherapy, both vitiligo and healthy skin showed
\end{abstract}

\section{KARGER}

(c) 2019 S. Karger AG, Basel

Ė-Mail karger@kargercoun

www.karger.com/drm an increase in MED and MI* values and a reduction of skin erythema compared to baseline $(p<0.05)$. MED-PF data showed a photoadaptation in $10(71.4 \%)$ vitiligo lesions and in $12(85.7 \%)$ healthy skin areas. The assessment of the $a^{*}$-PF showed a negative mean percentage value in all affected and unaffected skin areas. Conclusions: A short cycle of NBUVB phototherapy can induce photoadaptation in vitiligo by increasing the MED and reducing skin erythema after stimulation with SSR. This is most likely due to the physical filter function induced by ultraviolet radiation.

2019 S. Karger AG, Basel

\section{Introduction}

Vitiligo is the most frequent acquired pigmentary disease affecting approximately $1-3 \%$ of the general population [1].

The aetiopathogenesis of vitiligo is complex: genetic, inflammatory and immunological factors participate in the loss of function and destruction of skin melanocytes. Nevertheless, the specific contribution of each feature and the initial inducing factor should be still clarified [2, 3].

Dr. Mariachiara Arisi

Dermatology Department, University of Brescia, ASST Spedali Civili of Brescia P.le Spedali Civili 1

17'-25123 Bresciu (Ilaly)

V-Mail mariachiara.arisi @iamail.com 


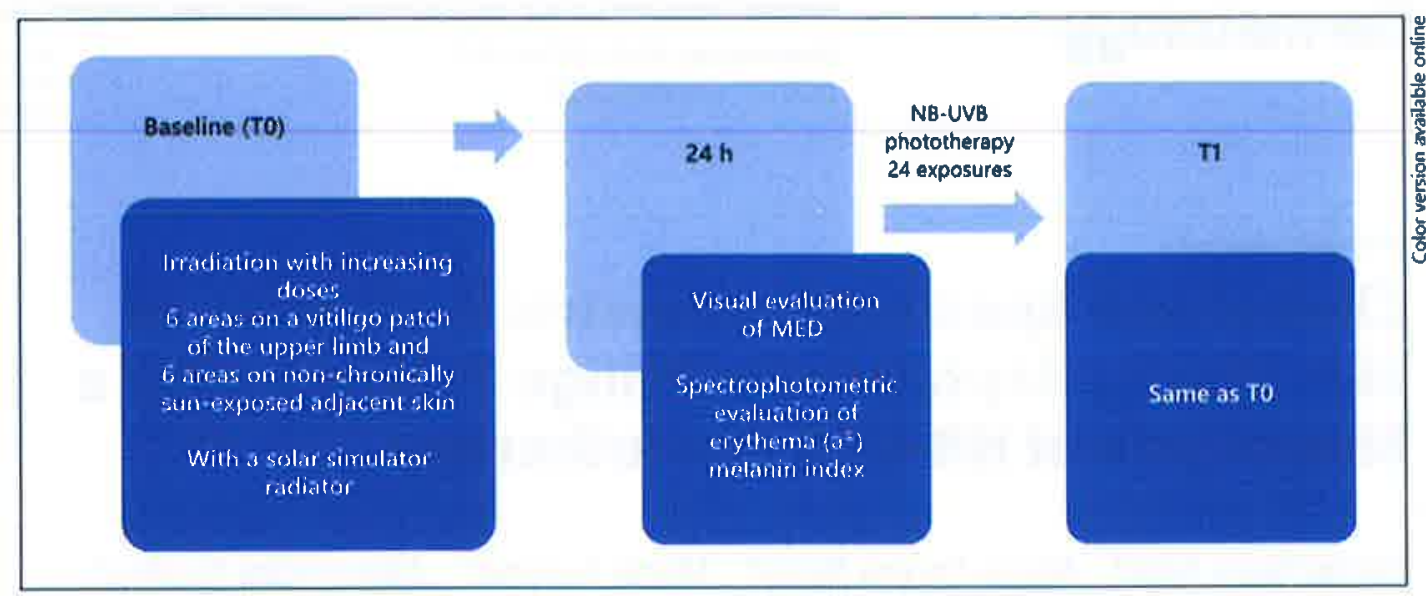

Flg. 1. Flowchart of Materials and Methods.

Therapeutic strategies for vitiligo include topical or systemic immunomodulant therapies whose aim it is to induce a partial or total pigmentation through melanocyte reactivation and melanin production.

Among systemic options, narrow-band ultraviolet $B$ (NB-UVB) phototherapy has shown similar efficacy to oral photochemotherapy (PUVA) with a lower risk of adverse events [4]; it represents the first-line treatment in non-segmental vitiligo [5].

However, during the first few weeks of phototherapy, it is difficult to detect a clinical response corresponding to an effective repigmentation of vitiligo lesions whereas a possible increase in pigmentation of healthy skin usually appears. Furthermore, a previous retrospective study showed that some patients with vitiligo treated with NBUVB did not develop phototoxicity in their depigmented skin despite increasing doses of UV radiation (UVR) [6]. Moreover, these patients underwent the phenomenon of photoadaptation or tolerance to NB-UVB radiation without repigmentation, a phenomenon frequently observed clinically during the course of phototherapy. Photoadaptation has usually been clinically measured by the assessment of the minimal erythema dose (MED) after phototesting with different UV wavebands [7], while only one work described the quantitative photoadaptive response to ultraviolet exposure in human skin using ultraviolet spectrophotometry [8]. Therefore, we designed a study with the purpose of measuring photoadaptation to a short cycle of NB-UVB phototherapy in vitiligo and healthy skin. The aim of our study was to evaluate spectrophotometric and clinical early modifications occur- ring after irradiation and to highlight possible subclinical parameters of therapeutic response to NB-UVB phototherapy.

\section{Materials and Mothods}

For further details, see the online supplementary material (see www.karger.com/doi/10.1159/000502853 for all online suppl. material) (Fig. 1) [9-14].

\section{Results}

Fourteen patients ( 2 males and 12 females; mean age: 43 years; range: $25-66$ ), affected by generalized vitiligo at baseline were enrolled in this study. Five (35.7\%) patients presented skin photoype II, 7 (50\%) patients photoype III and $2(14.3 \%)$ patients phototype V. Seven patients had a concomitant diagnosis of auto-immune hypothyroidism, and 3 patients reported a positive family history for vitiligo.

NB-UVB phototherapy was performed in all patients twice weekly for 12 weeks. The mean session number was 21.5 (range: 14-26), and the mean cumulative dose was $10.4 \mathrm{~J} / \mathrm{cm}^{2}$ (range: $5.3-13.75$ ).

\section{Clinical and Spectrophotometric Evaluation at Baseline (TO)}

The Kolmogorov-Smirnov test showed the absence of any normal distribution of data $(p<0.05)$.
Arisi et al. 
Fig. 2. Clinical assessment of minimal erythema dose (MED) at baseline in healthy skin (a) and vitiligo (b) and after NB-UVB phototherapy (c).
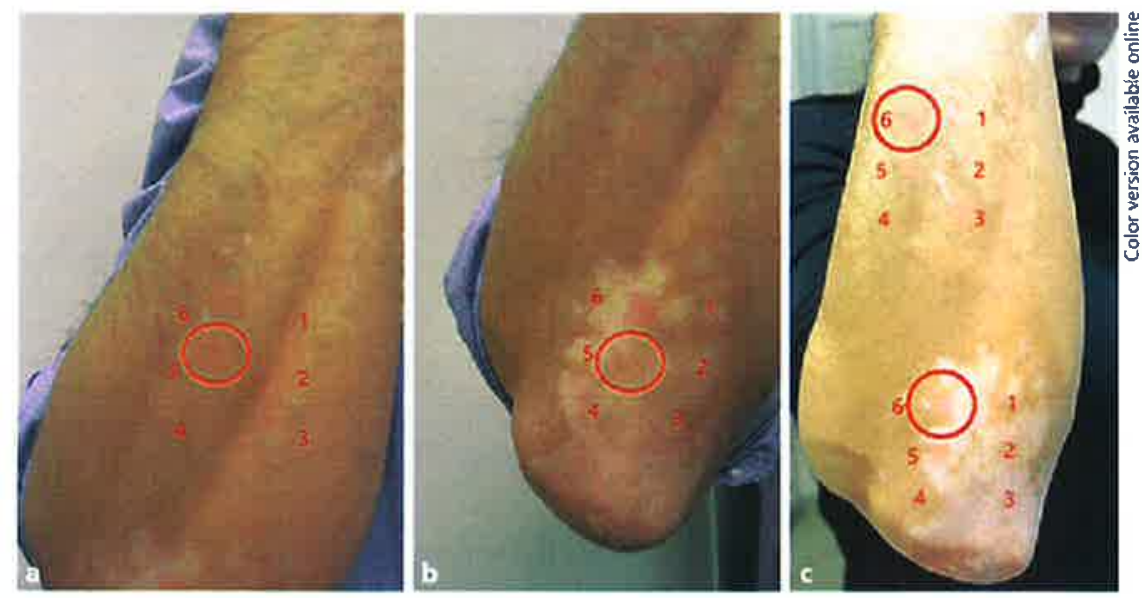

Table 1. Results (medians; minimum-maximum) of clinical and spectrophotometric evaluation in lesional and unaffected skin before and after NB-UVB phototherapy

\begin{tabular}{lllll}
\hline & Vitiligo T0 & Healthy skin T0 & Vitiligo T1 & Healthy skin T1 \\
\hline MED & 0.051 & 0.064 & 0.064 & 0.078 \\
& $(0.025-0.078)$ & $(0.032-0.098)$ & $(0.04-0.078)^{\mathrm{b}}$ & $(0.064-0.122)^{\mathrm{c}}$ \\
$\mathrm{a}^{*}$ value & 9.04 & 7.535 & 6.67 & 5.21 \\
& $(5.24-16.86)$ & $(4.31-12.06)$ & $(3.66-12.02)^{\mathrm{b}}$ & $(3.19-9.28)^{\mathrm{a}}$ \\
MI $^{*}$ & 0.75 & 1.376 & 0.77 & 1.622 \\
& $(0.28-1.39)$ & $(0.77-1.802)^{\mathrm{b}}$ & $(0.29-1.98)^{\mathrm{b}}$ & $(0.98-1.99)^{\mathrm{a}} \mathrm{c}$ \\
\hline
\end{tabular}

MED, minimal erythema dose; MI*, melanin index. " $p<0.05$ versus healthy skin at baseline; ${ }^{\mathrm{b}} p<0.05$ versus vitiligo at baseline; " $\rho<0.05$ versus vitiligo at $\mathrm{T} 1$.

At baseline, vitiligo lesions showed lower MED values and higher $\mathrm{a}^{*}$ values compared to healthy skin without reaching a statistical significance $(p=0.078 ; p=0.082)$ (Fig. 2a, b). Conversely, the melanin index (MI*) in affected skin areas was statistically significantly lower than in healthy $\operatorname{skin}(p<0.0002)$. (Table 1$)$.

No correlation was found between MED values at baseline and phototype, both in vitiligo $(p=0.153)$ and healthy $\operatorname{skin}(p=0.601)$, although a positive trend of relation was seen $(R=0.438$ and 0.153$)$.

\section{Clinical and Spectrophotometric Evaluation after $N B-U V B(T I)$}

After NB-UVB phototherapy, both vitiligo and healthy skin showed an increase in MED and MI* values and a reduction of skin erythema compared to baseline results $(p<0.05)$ (Table 1, Fig. 2c).

Photoadaptation in Vitiligo after NB-UVB Phototherapy
The comparison of clinical and spectrophotometric data in vitiligo lesions compared to unaffected skin confirmed lower MED and MI* values in vitiligo after phototherapy $(p=0.001 ; p<0.0002)$. $\mathrm{a}^{*}$ values remained higher in affected skin without reaching a statistical significance $(p=0.136)$.

The Kruskal-Wallis test, used to evaluate clinical and spectrophotometric feature variation after treatment, according to skin phototype, did not show any statistically significant result $(p=0.571)$. Linear regression revealed that disease duration (expressed in years) did not affect clinical or colorimetric results in vitiligo after phototherapy $(Y=X \times 0.000+0.064)$.

MED photoadaptation factor (MED-PF) data showed a photoadaptation in 10 (71.4\%) vitiligo lesions and in 12 (85.7\%) healthy skin areas (Fig. 3a). The assessment of the $a^{*}$ photoadaptation factor ( $\left.a^{*}-\mathrm{PF}\right)$ showed a negative mean percentage value in all affected and unaffected skin 
Fig. 3. Photoadaptation or percentage change in minimal erythema dose (MED) (a) and $\mathrm{a}^{*}(\mathrm{~b})$ in vitiligous and normal skin after NB-UVB phototherapy.
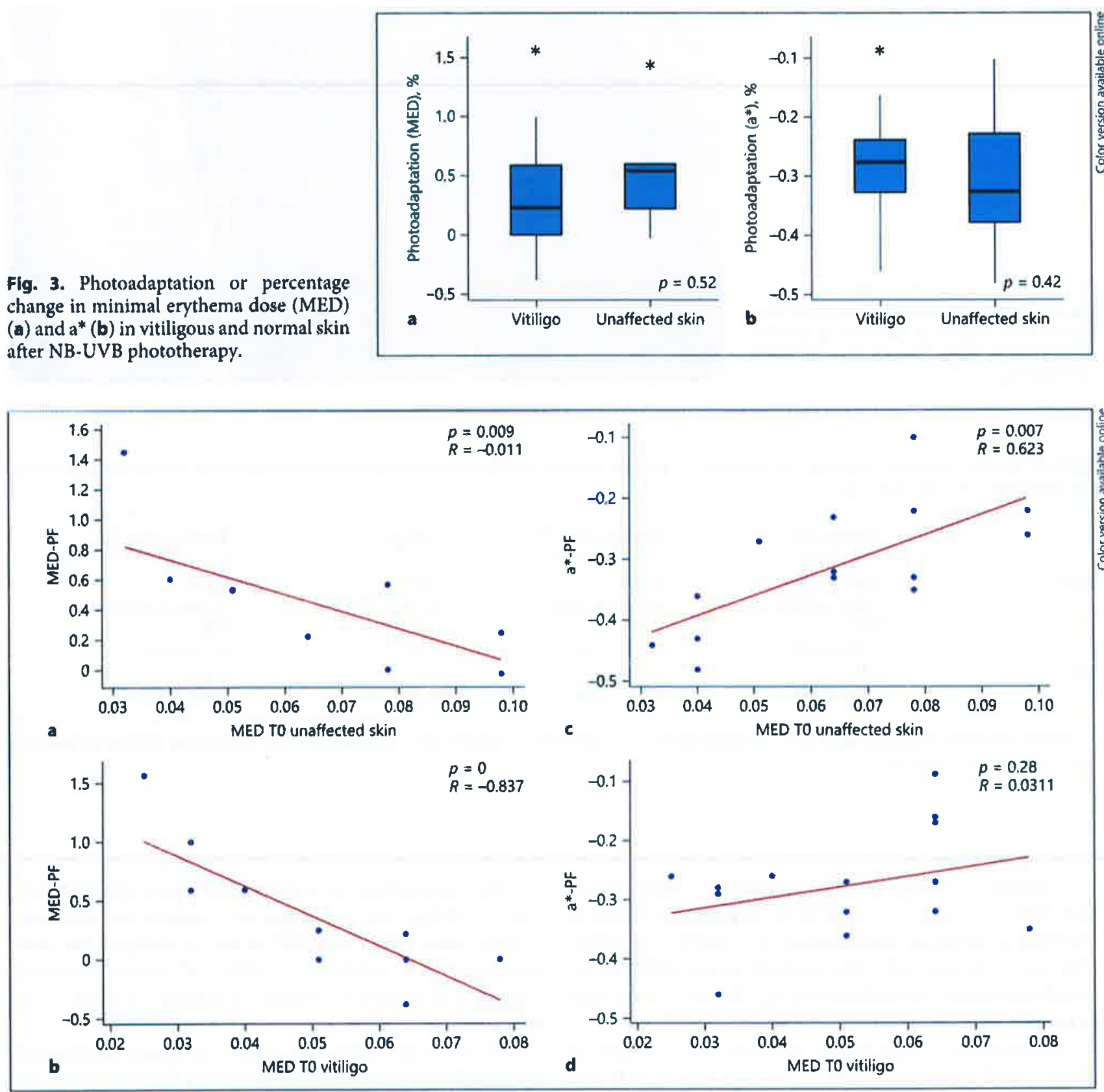

Fig. 4. Correlation between baseline minimal erythema dose (MED) and photoadaptation factors (PF) in vitiligo $(b, d)$ and unaffected skin $(a, c)$.

areas (Fig. 3b). Photoadaptation or percentage change in MED and a* was not statistically different between vitiliginous and normal skin $(p=0.52$ and 0.42$)$.

A negative significant correlation between baseline MED was seen and MED-PF both in vitiligo $(p=0 ; R=$
$-0.837)$ and healthy skin $(p=0.009 ; R=-0.011)$ (Fig. 4a, b) while a positive correlation was seen if analysing baseline MED and $\mathrm{a}^{*}$-PF, in affected $(p=0.28 ; R=$ $0.0311)$ and unaffected skin $(p=0.007 ; R=0.623)$ (Fig. 4c, d). 
If comparing skin phototype to MED-PF in vitiligo and healthy skin, a negative correlation was seen without reaching a statistical significance $(p=0.886$ and $0.73 ; R=$ -0.042 and -0.043$)$. The same results were found when correlating $\mathrm{a}^{*}$-PF to skin phototype in vitiligo $(p=0.84$; $R=0.056)$ and healthy skin $(p=0.133 ; R=0.422)$ but a positive trend of correlation was seen.

\section{Discussion/Conclusion}

NB-UVB phototherapy is the preferred therapy for active and generalized vitiligo which has shown higher efficacy rates compared to other treatment modalities such as photochemotherapy (PUVA) $[5,15,16]$.

Within the first 3 months, approximately $80 \%$ of subjects show a response to therapy. However, the period of treatment necessary to obtain a complete repigmentation is generally longer: $60 \%$ of patients require at least 1 year of phototherapy for a repigmentation of at least $50 \%$ of skin lesions, while a 3-month therapy results in moderate responses (up to $25 \%$ of repigmentation) [17]. Photoadaptation has been previously described in vitiligo and psoriasis $[7,18,19]$. It was first defined by $\mathrm{Oh}$ et al. [20] as adaptive and diminished skin responses to equivalent doses of radiation. Thus, subsequent equivalent UVR doses do not have the same effect on the skin as the first dose. Photoadaptation has been described in different Fitzpatrick skin phototypes and is thought to be due to both pigmentary and non-pigmentary influences [19, 21]. Studies have indicated that principal mechanisms involved include hyperkeratosis, acanthosis, melanogenesis as well as an unknown factor yet to be identified which may be due to vascular reactivity, DNA repair or an immune-related process [8, 21-25]. A study from Hexsel et al. [18] analysed cyclobutene pyrimidine dimer production and repair in vitiligo and healthy skin after NB-UVB exposure showing that more cyclobutene pyrimidine dimers are repaired in photoadapting vitiligo lesions. Interestingly, the same work reported a photoadaptation also in non-exposed healthy skin but not in non-treated vitiligo lesions, suggesting a kind of systemic effect of pigmentation.

Photoadaptation has been usually assessed clinically on the basis of MED changes after UV exposure $[7,18,21$, 26]. However, clinical inspection alone may be inadequate as it is limited by interrater reliability and by the impossibility of identifying variations not visible to the naked eye. So, objective and comparable instrumental tools are needed.

Photoadaptation in Vitiligo after NB-UVB Phototherapy
In the present study we performed a spectrophotometer evaluation to highlight skin colour changes after NBUVB phototherapy both in vitiligo and unaffected surrounding skin, by analysing two main parameters such as erythema $\left(\mathrm{a}^{*}\right)$ and melanin index $\left(\mathrm{MI}^{*}\right)$, and we considered the first as an additional sign of photoadaptation in addition to MED assessment.

The spectrophotometer is an instrument capable of making objective measurements of a visible light spectrum reflected by an object under examination, analysing chromatic and shape parameters of the light frequency band. In dermatology, it is used to acquire quantitative and specific data on the chromatic components of the skin, in basal and experimental conditions, which are mostly clinically undetectable [27].

In our series, clinical evaluation of MED highlighted increased values after phototherapy in both affected and healthy skin even if vitiligo patches preserved lower MED values as baseline. These results were supported by the spectrophotometric evaluation of baseline MED skin area which showed a reduction of $\mathrm{a}^{*}$ values both in vitiligo and healthy skin.

MI* evaluation revealed much lower pigmentation values in vitiligo skin at baseline and after phototherapy, in line with the absence of melanin pigment typical of vitiligo. MI* values were increased by NB-UVB not only in healthy skin areas, but also in non-pigmented vitiligo areas, even though reaching lower values in the latter.

Clinical evaluation of our patients showed positive MED-PF results in only $10(71.4 \%)$ vitiligo lesions and in $12(85.7 \%)$ healthy skin areas. Interestingly, the assessment of the $\mathrm{a}^{*}$-PF showed a negative mean percentage value in all affected and unaffected skin areas, confirming a greater sensitivity of the instrumental evaluation of skin photoadaptation to NB-UVB.

These results show how a short cycle of phototherapy is not concretely able to induce a clinically evident repigmentation in vitiligo, but it can still exert a beneficial effect of photoadaptation demonstrated by the increase in the MED and reduction of the skin erythema after stimulation with solar simulated radiation. Reduction of $\mathrm{a}^{*}$ values in pathological and healthy skin is most likely due to the physical filter function of the increased thickness of the stratum corneum induced by UVR.

Interestingly, we noted how subjects with lower MED values at baseline showed higher rates of photoadaptation in terms of MED-PF and $\mathrm{a}^{*}-\mathrm{PF}$. These results suggest a sort of functional reserve of photoadaptation typical of subjects with reduced MED values, and that is common to healthy and vitiligo skin.

Dermatology 2019;235:509-515 DOI: $10.1159 / 000502853$ 
Moreover, we reported an $\mathrm{MI}^{*}$ increase in vitiligo after phototherapy without a clinically visible repigmentation. We can conceive that this result is obviously not due to a melanin augmentation but to an increment and retention of oxidized protein substances in keratinocytes which are able to absorb in the same spectral range of melanin in the sensitivity range of the spectrophotometer $(400-700 \mathrm{~nm})$.

This seems to contribute to the photoadaptation process in vitiligo mediated by short-term NB-UVB exposure, and it may have a protective effect both against the risk of sunburns and the risk of UV-induced carcinogenesis.

However, in our series, photoadaptation or percentage change in MED and a* values was not statistically different between vitiliginous and normal skin. This result can be explained by the fact that physiological processes involved in skin photoadaptation to UVR do not differ in normal or pathological skin.

To the best of our knowledge, this is the first work in which photoadaptation of vitiligo has been demonstrated by clinical and spectrophotometric evaluation. We do think that a better knowledge in vitiligo photoadaptation may be useful in constructing phototherapy dosing schedules. Awareness of the early effects of NB-UVB in vitiligo lesions would avoid the use of under-or overlabelled protocols, increasing the probability of lesion repigmentation and minimizing episodes of burning. Finally, an optimized phototherapy dosing would decrease the number of patients' hospital accesses and costs.
Nevertheless, the main limitations of our study are that we did not perform a phototest also on untreated vitiligo and healthy skin areas as controls to those treated with NB-UVB, and we were unable to identify any possible association between MED or spectrophotometric parameters and skin phototype probably due to the small sample size.

Further research on wider case series would be able to detect the variables that predict the photoadaptive response in vitiligo.

\section{Key Message}

A short cycle of narrow-band ultraviolet B phototherapy can induce photoadaptation in vitiligo by increasing the minimal erythema dose and reducing skin erythema after stimulation with solar simulated radiation. This is most likely due to the physical filter function induced by ultraviolet radiation.

\section{Statement of Ethics}

Subjects have given their written informed consent. The study protocol has been approved by the research institute's committee on human research.

\section{Disclosure Statement}

The authors have no conflicts of interest to declare.

\section{References}

1 Exzedine K, Eleftheriadou V, Whitton $M$, van Geel N. Vitiligo. Lancet, 2015;386(9988):7484.

2 Spritz RA, Andersen (iH. Genetics of vitiligo. Detmatol Clin, 2017:35(2):245-55.

3 Mohammed GF, Ciomaa AH, Al-Dhubaibi MS. Highlights in pathogenesis of Vitlligo. World J Clin Cases, 2015;3(3):221-30.

4 Ortel B, Petrovic-Rusic V, Calzavara-Pinton PG. Phototherapeutic options for vitiligo. In Krutmanu J, Honigsmann $H$, Hlmets CA, Bergstresser PR, editors. Dermatological phototherapy and photodiagnostic methods. London: Springer; 2009.

5 Westerhof W, Nleuweboer-Krobotova L. Treatment of vitiligo witl UV-B radiation vs topical psoralen plus UV-A. Arch Dermatol. 1997:133(12):1525-8.

6 Hamzavi I, Deleon S, Yue K, Murakawa G. Repigmentation does not affect tolerance to NB-UVB light in patients with vitiligo. Photodermatol Photoimmunol Photomed, 2004; 20:117.
7 Gonzalez S, Hegyi V, Baqer A, Kollias N. Development of cutancous tolerance to ultraviolet B during ultraviolet B phototherapy for psoriasis. Photodermatol Photoimmunol photomed. 1996; 12(2):73-8.

8 Hennessy A, Oh C. Rees J. Diffey B. The photoadaptive response to ultraviolet exposure in human skin using ultraviolet spectrophotometry. Photodermatol Photoiminumal Photomed, 2005:21(5):229-33.

9 Madigan L.M. Al-jamal M, Hamzavi I, Exploring the gaps in the evidence-based application of narrowband UVB for the treatment of vitiligo. Photodernatol Photoimmunol Photomed. 2016;32(2):66-80.

10 Otman SG, Edwards C, Gambles B, Anstey AV. Validation of a semiautonated method of minimal erythema dose testing for narrow. band ultraviolet B plototherapy. Br I Dermittol. 2006;155(2):416-21.
11 Commission International de L'Eclairage (CIE). Colorimetry - technical report. CIE publication No. 15. 3rd ed. Viennit: Bureau Central de la C.IE, 2004.

12 Miller SA, Coelho SG, Zmudzka BZ, et al. Dy. namics of pigmentation induction by repeated UV exposures: dose, dose interval and ultraviolet spectrum dependence. $\mathrm{Br}$ I Dexmatol. $2008 ; 159(4): 921-30$.

13 Wagner JK, Jovel C, Norton HL, Parra EJ, Shriver MD. Comparing quantitative measures of erythenu, pigmentation and skin response using reflectometry. Pigment Cell Res. 2002;15(5):379-84.

14 Choi KW, Kim KH, Kim YH. Comparative study of the gross interpretation of pholotesting and objective measurement with using a spectrophotometer for patients with psoriasis and vitiligo treated with narrow-band UVB. Ann Dermatol. 2009;21(2):136-41. 
15 Taieb A, Alomar A, Boelum $M$, et al, Guide lines for the management of vitiligo. The European Dermatology Forum consensus. $\mathrm{Br}$ I Dermatul. 2013;168(1):5-19.

16 Boniface K, Seneschal J, Picardo M, Taieb A. Vitiligo: focus on clinical aspects, immunopathogenesis, and therapy. Clin Rev Allergy Inmunol. 2018:54(1):52-67.

17 Bae JM, Jung HM, Hong BY, Lee JH, Choi WJ. Lee $\mathrm{JH}$, et al. Phototherapy for vitiligo: a systematic review and meta-analysis. JAMA Dermatol. 2017;153(7):666-74

18 Hexsel CL, Mahmoud BH, Mitchell D, Rivard J, Owen M, Strickland FM, et al. A clinical trial and molecular study of photoadaptation in vitiligo. Br I Dermatol. 2009;160(3):534-9.

19 Palmer RA, Aquilina S, Milligan PJ, Walker SL, Hawk JL, Young AR, Photoadaptation during narrowband ultraviolet-B therapy is independent of skin type: a study of $352 \mathrm{pa}-$ tients. J Invest Dermatol, 2006;126(6):125663.
20 Oh C, Hennessy A, Ha T, Bisset Y, Diffey B, Rees JL. The time course of photoadaptation and pigmentation studied using a novel method to distinguish pigmentation from erythema. I lrivest Dermatol. 2004;123(5):965-72.

21 Rivard I, Hexsel C, Owen M, Strickland FM, Lim HW. Hamzavi I. Photoadaptation of vitiliginous skin to targeted ultravlolet B phototherapy, Photodermatol Photoimmunol Photomed. 2007;23(6):258-60.

22 Lock-Andersen J, Therkildsen P, de Fine Olivarius $\mathrm{F}$, et al. Epidermal thickness, skin pigmentation and constitutive photosensitivity. Photodernatol Plotoimmunol photomed. 1997;13(4):153-8.
23 Bataille V, Bykov V], Sasieni P, Harulow S, Cuzick J, Hemminki K. Photoadaptation to ultraviolet (UV) radiation in vivo: photoproducts in epidermal cells following UVB therapy for psoriasis. Br I Dermatol. 2000; 143(3):477-83.

24 Hamzavi I. Photoadaptation: a path toward rational phototherapy protocols. J Invest Dermatol. 2006:126(10):2156-8.

25 Mitchell DL, Greinert R, de Gruijl FR, et al. Bffects of chronic low-dose ultraviolet B radiation on DNA damage and repair in mouse skin. Cancer Res. 1999;59:2875-84.

26 Darné S, Stewart LC, Farr PM, Hampton PJ. Investigation of cutaneous photoadaptation to narrow-band ultraviolet $\mathrm{B}$. $\mathrm{Br}$ I Dermatol. 2014;170(2):392-7.

27 Takiwaki H. Measurement of skin color: practical application and theoretical considerations. J Med Invest. 1998;44:121-6. 
